07

\title{
Возрастание мощности колебаний тока в полупроводниковой сверхрешетке с учетом межминизонного туннелирования
}

\author{
(c) А.О. Сельский
}

Саратовский государственный университет им. Н.Г. Чернышевского, Саратов, Россия

E-mail: feanorberserk@gmail.com

Поступило в Редакцию 29 сентября 2017 г.

Рассматривается полная мощность колебаний тока, протекающего через полупроводниковую сверхрешетку, при различных значениях ширины запрещенной зоны между первой и второй минизонами. Показано, что при уменьшении величины запрещенной зоны, когда вероятность межминизонного туннелирования возрастает, полная мощность колебаний тока увеличивается при некоторых значениях напряжения, приложенного к сверхрешетке.

DOI: $10.21883 /$ PJTF.2018.09.46065.17062

Объектом исследования в настоящей работе является гетероструктура, состоящая из чередующихся слоев (ширина слоя, как правило, не превышает нескольких десятков нанометров) различных полупроводниковых материалов с близкими периодами кристаллической решетки, - полупроводниковая сверхрешетка [1,2]. Сверхрешетки служат удобной моделью для изучения различных квантово-механических эффектов [3,4]. Не меньший интерес вызывает коллективная динамика электронов при приложении к сверхрешетке постоянного напряжения, поскольку в этом случае в структуре могут образовываться пространственно-временны́е электронные структуры, называемые доменами (по аналогии с доменами в диоде Ганна $[5,6])$. Прохождение доменов через полупроводниковую сверхрешетку приводит к возникновению колебаний тока, протекающего через структуру, частота которых составляет, как правило, несколько десятков $\mathrm{GHz}[7,8]$. Следует отметить, что в настоящее время все больше внимания уделяется рассмот- 
рению транспорта электронов через полупроводниковую сверхрешетку с позиций нелинейной динамики, что позволяет выявлять и объяснять различные эффекты, наблюдающиеся в исследуемой системе [7-11].

Обычно при моделировании и изготовлении сильносвязанных сверхрешеток расстояние между минизонами задается достаточно большим, чтобы можно было пренебречь межминизонным туннелированием Ландау-Зинера $[7,8,12]$. В этом случае существенно упрощаются как описание транспорта зарядов в сверхрешетках, так и интерпретация экспериментальных данных. Однако в ряде работ было показано, что учет межминизонного туннелирования сильно изменяет групповую динамику электронов в сверхрешетках, в частности спектральные характеристики колебаний тока, протекающего через сверхрешетку $[13,14]$. В настоящей работе рассмотрено влияние межминизонного туннелирования на полную мощность колебаний тока.

Типовой моделью при описании процессов транспорта заряда в полупроводниковой сверхрешетке является система уравнений, включающая в себя уравнение непрерывности, уравнение Пуассона и выражение для плотности тока с учетом дрейфовой скорости электрона [2,9]:

$$
\begin{gathered}
e \frac{\partial n}{\partial t}=\frac{\partial J}{\partial x}, \\
\frac{\partial F}{\partial x}=\frac{e}{\varepsilon_{0} \varepsilon_{r}}\left(n-n_{D 0}\right), \\
J=e n v_{d}(\bar{F}),
\end{gathered}
$$

где $t$ обозначает время, а координата $x$ соответствует направлению движения электронов в полупроводниковой сверхрешетке. Величины $n(x, t), F(x, t)$ и $J(x, t)$ определяют концентрацию, напряженность электрического поля и плотность тока соответственно. Параметры $\varepsilon_{0}, \varepsilon_{r}=12.5$ соответствуют абсолютной и относительной диэлектрическим проницаемостям; $n_{D 0}=3 \cdot 10^{22} \mathrm{~m}^{-3}-$ равновесная концентрация электронов; $v_{d}$ - дрейфовая скорость электрона, вычисленная для среднего значения напряженности электрического поля $\bar{F} ; e>0-$ заряд электрона.

В случае отсутствия наклонного магнитного поля зависимость дрейфовой скорости от напряженности электрического поля, входящая в уравнение (1), может быть вычислена аналитически с помощью 

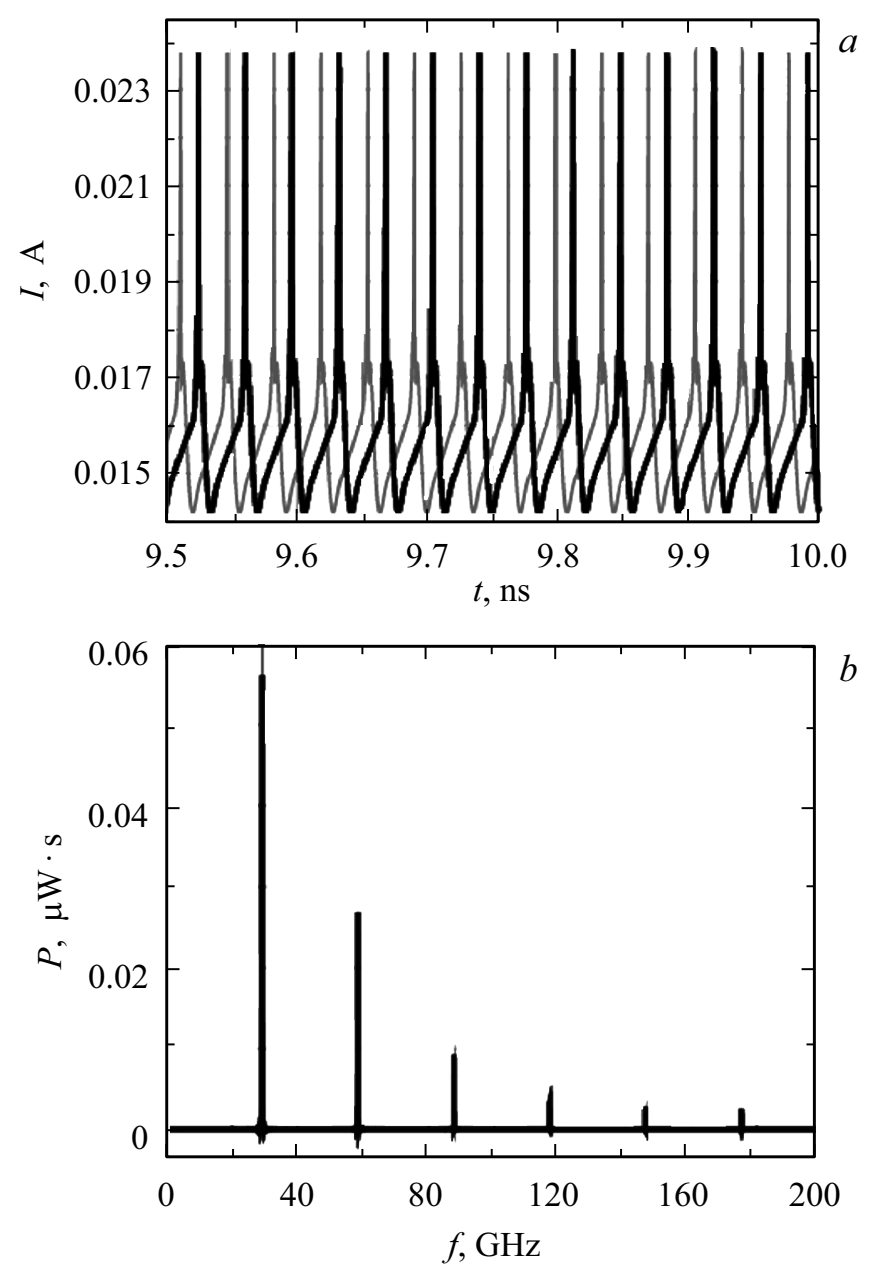

Рис. 1. $a, c$ - реализации тока, протекающего через полупроводниковую сверхрешетку, $b, d-$ спектральные плотности мощности колебаний тока, протекающего через полупроводниковую сверхрешетку. Значения напряжения, приложенного к полупроводниковой сверхрешетке: $V=0.4(a, b)$ и $0.65 \mathrm{~V}(c, d)$. Черная линия построена для значения ширины запрещенной зоны $E_{g}=200 \mathrm{meV}$, серая линия - для значения $E_{g}=130 \mathrm{meV}$.

4* Письма в ЖТФ, 2018, том 44, вып. 9 

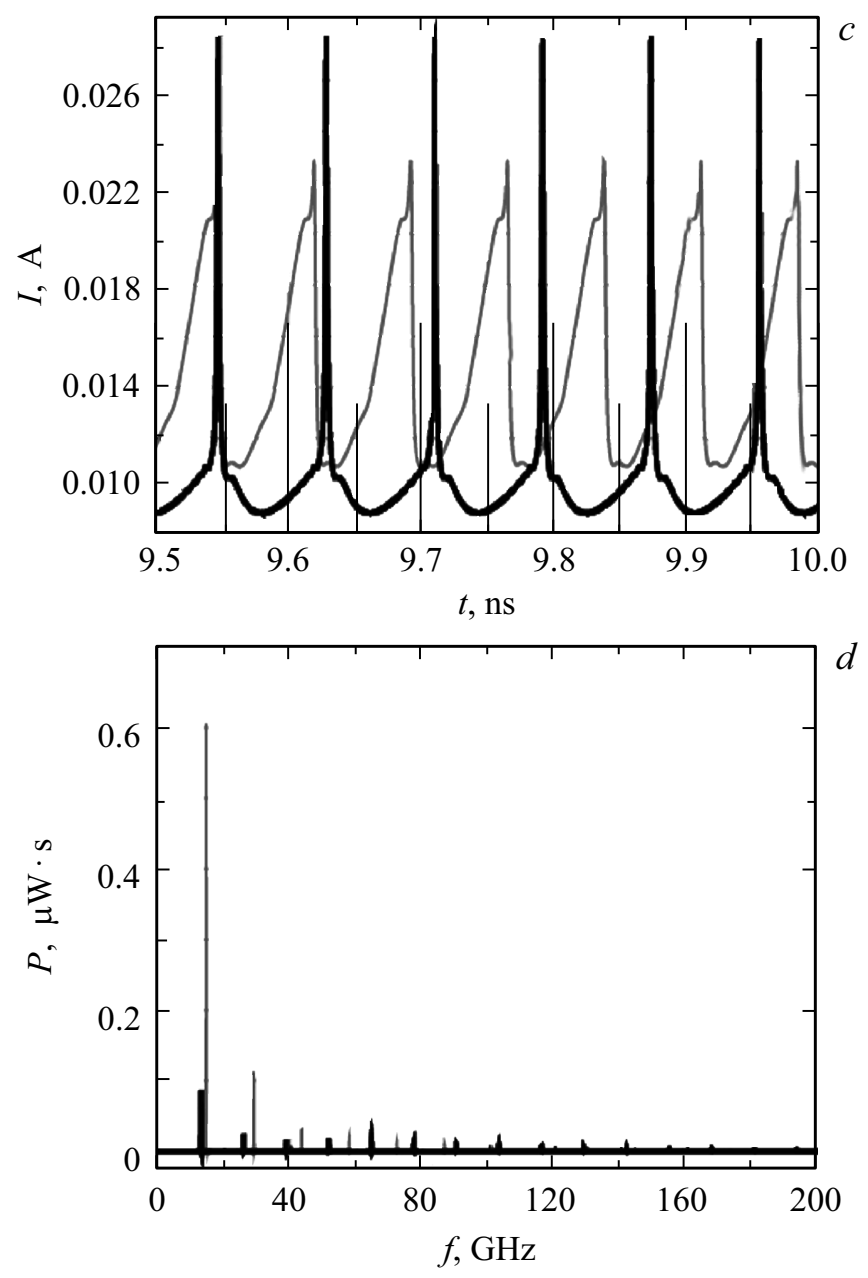

Рис. 1 (продолжение). 
формулы Эсаки и Тсу [4]

$$
v_{d}=\frac{\Delta d}{2 \hbar} \frac{\omega_{\mathrm{B}} \tau}{\left(\omega_{\mathrm{B}} \tau\right)^{2}+1},
$$

где $\Delta-$ ширина первой минизоны, $d-$ период сверхрешетки, $\tau-$ эффективное время рассеяния электрона, $\omega_{\mathrm{B}}=e F d / \hbar-$ частота колебаний Блоха. Для учета возможности туннелирования между первой и второй минизонами применялся подход, описанный в $[15,16]$. В соответствии с этими работами дрейфовая скорость с учетом межминизонного туннелирования определяется следующим образом:

$$
\begin{gathered}
v_{d, \text { mod }}=v_{d}(1-T(F))+T(F) v_{d, \text { free }}, \\
v_{d, \text { free }}=\frac{e F \tau}{2 m^{*}},
\end{gathered}
$$

где $m^{*}-$ эффективная масса электрона в полупроводнике. В данном случае $v_{d, f r e e}-$ дрейфовая скорость электрона во второй минизоне, рассчитанная в приближении свободного электрона. Вероятность межминизонного туннелирования $T(F)$ определяется в соответствии с работами [15-17] как

$$
T(F)=\exp \left(\frac{m^{*} d E_{g}^{2}}{4 \hbar^{2}|e F|}\right),
$$

где $E_{g}$ - ширина запрещенной зоны между первой и второй минизонами. Из уравнений (3)-(5) можно видеть, что при увеличении вероятности межминизонного туннелирования дрейфовая скорость возрастает, что существенным образом влияет на групповую динамику электронов в полупроводниковой сверхрешетке. При ширине запрещенной зоны $E_{g} \rightarrow \infty$ формула (3) преобразуется в формулу (2).

В работе [14] было показано, что в присутствии наклонного магнитного поля увеличение вероятности межминизонного туннелирования приводит к увеличению мощности старших гармоник колебаний тока, протекающего через полупроводниковую сверхрешетку. В настоящей работе рассматривается более простой случай, когда магнитного поля нет. В этом случае при уменьшении ширины запрещенной зоны между первой и второй минизонами можно наблюдать существенное увеличение старшей гармоники (рис. $1, d$ ). Важно отметить, что при

Письма в ЖТФ, 2018, том 44, вып. 9 

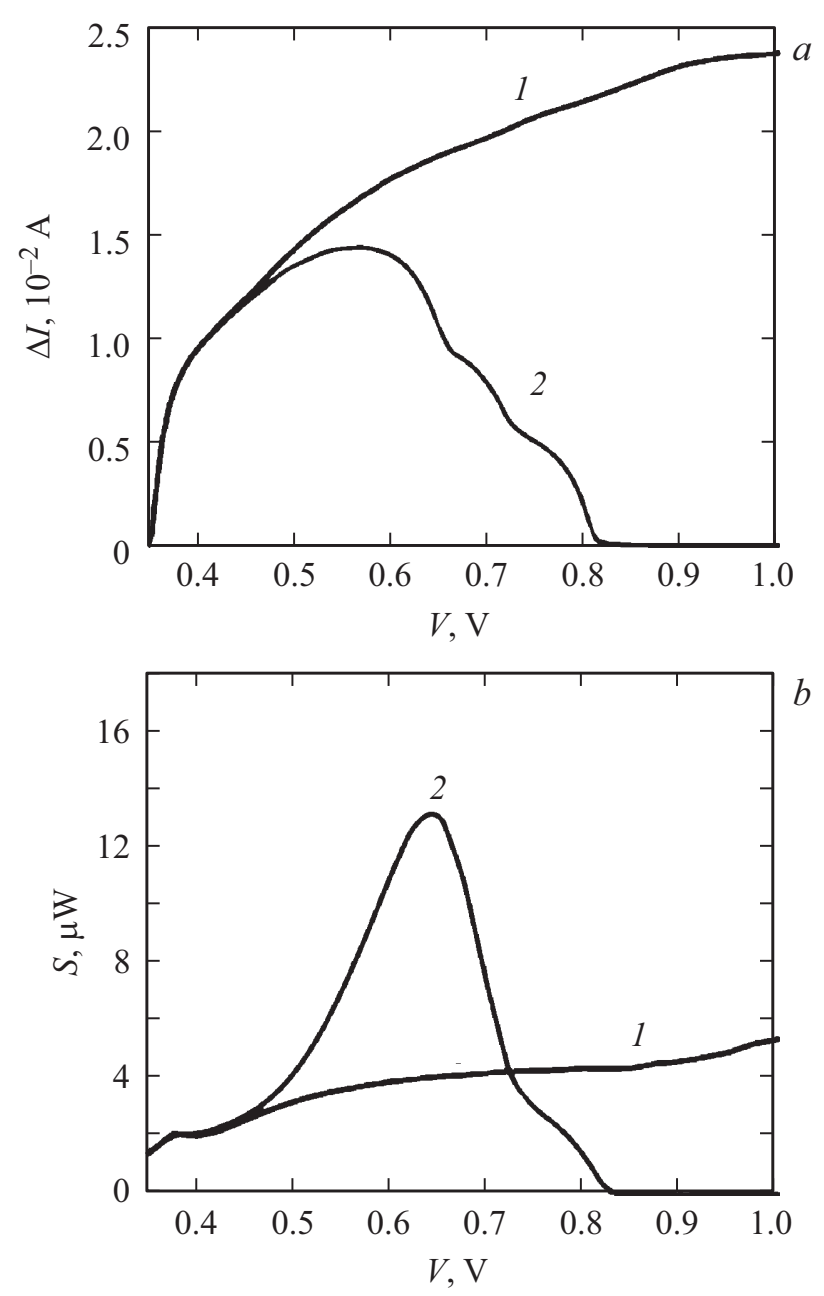

Рис. 2. Зависимости амплитуды колебаний тока, протекающего через полупроводниковую сверхрешетку, от напряжения, приложенного к ней $(a)$, и полной мощности колебаний от напряжения $(b)$ при значениях ширины запрещенной зоны $E_{g}=200(1)$ и $130 \mathrm{meV}(2)$.

Письма в ЖТФ, 2018, том 44, вып. 9 
более низких значениях напряжения (рис. 1,b) спектральные плотности мощности колебаний тока при различных значениях ширины запрещенной зоны почти идентичны. Это свидетельствует о том, что увеличение мощности старшей гармоники связано с межминизонным туннелированием, так как при низких напряжениях не наблюдается значений напряженности электрического поля, достаточных для существенного увеличения вероятности туннелирования в соответствии с формулой (5). На рис. $1, a$ и $c$ можно сравнить вид колебаний тока при различных значениях напряжения и ширины запрещенной зоны, определяющих вероятность межминизонного туннелирования. Можно видеть, что для высоких значений напряжения вид колебаний существенно изменяется, амплитуда уменьшается, тогда как пики тока расширяются.

На рис. 2 приведены зависимости амплитуды колебаний тока и полной мощности колебаний от напряжения при различных значениях ширины запрещенной зоны. Полная мощность рассчитывалась с помощью интегрирования спектральной плотности мощности по всем частотам. Из рисунка видно, что для ширины запрещенной зоны $E_{g}=200 \mathrm{meV}$, когда туннелированием можно пренебречь, амплитуда и полная мощность колебаний тока монотонно возрастают с ростом напряжения. При ширине запрещенной зоны $E_{g}=130 \mathrm{meV}$ амплитуда с некоторого значения напряжения начинает уменьшаться, пока не становится равной нулю, что означает полное подавление колебаний тока. Полная мощность, так же как и амплитуда, проходит через максимум, после чего уменьшается до нуля. Важно отметить, что амплитуда колебаний тока при ширине запрещенной зоны $E_{g}=130 \mathrm{meV}$ меньше, чем при $E_{g}=200 \mathrm{meV}$, для всех значений напряжения, приложенного к полупроводниковой сверхрешетке. В то же время полная мощность существенно выше при ширине запрещенной зоны $E_{g}=130 \mathrm{meV}$ для некоторого диапазона напряжений $0.5-0.7 \mathrm{~V}$.

Таким образом, в работе показано, что влияние межминизонного туннелирования приводит к изменению колебаний тока, протекающего через полупроводниковую сверхрешетку. Амплитуда колебаний с ростом вероятности туннелирования уменьшается вплоть до нуля. Вместе с тем изменяется форма колебаний, что ведет к росту старшей гармоники в спектральной плотности мощности. Этот рост приводит к появлению максимума полной мощности,

Письма в ЖТФ, 2018, том 44, вып. 9 
предшествующего подавлению колебаний. Величина максимума полной мощности существенно выше, чем значение полной мощности для случая, когда межминизонным туннелированием можно пренебречь.

Работа выполнена при поддержке Российского фонда фундаментальных исследований (проект № 16-32-00182).

\section{Список литературы}

[1] Шик А.Я. // ФТП. 1974. Т. 8. В. 10. С. 1841-1864.

[2] Wacker A. // Phys. Rep. 2002. V. 357. P. 1-111.

[3] Келдыш Л.В. // ФТТ. 1962. Т. 4. В. 8. С. 2265-2267.

[4] Esaki L., Tsu R. // IBM J. Res. Develop. 1970. V. 14. P. 61-65.

[5] Scheuerer R., Schomburg E., Renk K.F., Wacker A., Schöll E. // Appl. Phys. Lett. 2002. V. 81. P. 1515-1517.

[6] Eisele H., Khanna S.P., Lineld E.H. // Appl. Phys. Lett. 2010. V. 96. P. 072101.

[7] Greenaway M.T., Balanov A.G., Schöll E., Fromhold T.M. // Phys. Rev. B. 2009. V. 80. P. 205318.

[8] Fromhold T.M., Patanè A., Bujkiewicz S., Wilkinson P.B., Fowler D., Sherwood D., Stapleton S.P., Krokhin A.A., Eaves L., Henini M., Sankeshwar N.S., Sheard F.W. // Nature. 2004. V. 428. P. 726-730.

[9] Selskii A.O., Koronovskii A.A., Hramov A.E., Moskalenko O.I., Alekseev K.N., Greenaway M.T., Wang F., Fromhold T.M., Shorokhov A.V., Khvastunov N.N., Balanov A.G. // Phys. Rev. B. 2011. V. 84. P. 235311.

[10] Bonilla L.L., Teitsworth S.W. Nonlinear wave methods for charge transport. Wiley-VCH Verlag GmbH \& Co. KGaA, Weinheim, 2010. $287 \mathrm{p}$.

[11] Hramov A.E., Makarov V.V., Koronovskii A.A., Kurkin S.A., Gaifullin M.B., Alexeeva N.V., Alekseev K.N., Greenaway M.T., Fromhold T.M., Patané A., Kusmartsev F.V., Maksimenko V.A., Moskalenko O.I., Balanov A.G. // Phys. Rev. Lett. 2014. V. 112. P. 116603.

[12] Alexeeva N., Greenaway M.T., Balanov A.G., Makarovsky O., Patané A., Gaifullin M.B., Kusmartsev F., Fromhold T.M. // Phys. Rev. Lett. 2012. V. 109. P. 024102.

[13] Сельский А.О., Короновский А.А., Москаленко О.И., Храмов А.Е., Fromhold T.M., Greenaway M.T., Баланов А.Г. // ЖТФ. 2015. Т. 85. В. 4. C. 62-66.

[14] Balanov A.G., Koronovskii A.A., Moskalenko O.I., Selskii A.O., Hramov A.E. // Phys. Wave Phenom. 2015. V. 23. P. 28-34.

Письма в ЖТФ, 2018, том 44, вып. 9 
[15] Hardwick D.P.A., Naylor S.L., Bujkiewicz S., Fromhold T.M., Fowler D., Patané A., Eaves L., Krokhin A.A., Wilkinson P.B., Henini M., Sheard F.W. // Physica E. 2006. V. 32. P. 1-2.

[16] Hardwick D.P.A. Quantum and semiclassical calculations of electron transport through a stochastic system. PhD thesis. Nottingham: University of Nottingham, 2007. $223 \mathrm{p}$.

[17] Zener C. // Proc. Roy. Soc. Lond. A. 1934. V. 145. P. 523-529.

Письма в ЖТФ, 2018, том 44, вып. 9 